\title{
Nível de exposição ao Mycobacterium tuberculosis em estudantes de medicina de uma universidade do sul de Minas
}

\author{
Level of exposure to Mycobacterium tuberculosis in medical \\ students at a university in southern Minas
}

\section{Eugênio Fernandes de Magalhães', Claudinei Leôncio Beraldo², Júlio Pereira Costa Júnior ${ }^{3}$, João Paulo Cheregatti Amaral'3, Danilo Giffoni de Melo Morais Mata ${ }^{3}$, Bruno Leôncio de Moraes Beraldo ${ }^{4}$}

\begin{abstract}
Magalhães EF, Beraldo CL, Costa Júnior JP, Amaral JPC, Mata DGMM, Beraldo BLM. Nível de exposição ao Mycobacterium tuberculosis em estudantes de medicina de uma universidade do sul de Minas/Level of exposure to Mycobacterium tuberculosis in medical students at a university in southern Minas. Rev Med (São Paulo). 2011 abr.-jun.;90(2):94-9.
\end{abstract}

RESUMO: Objetivos. Verificar o nível de exposição ao Mycobacterium tuberculosis e os fatores de risco associados, utilizando o teste tuberculínico como marcador para tuberculose latente em universitários do curso de medicina da UNIVÁS em Pouso Alegre, MG, Brasil. Métodos. Estudo transversal com 120 estudantes divididos em três grupos: básico, intermediário e internato. Informacões relativas ao gênero, faixa etária, presença de cicatriz BCG, segunda dose de BCG, contato prévio com portador de tuberculose e história pessoal de tuberculose foram obtidas por meio de questionário. O teste tuberculínico foi realizado por profissional habilitado e foi definido como positivo quando a enduração foi maior de $5 \mathrm{~mm}$. Resultados. A prevalência de expostos ao Mycobacterium tuberculosis foi de $28,3 \%$, sendo mais frequente nos alunos do internato. Observou-se uma maior positividade no gênero feminino $(p>0,05)$. O teste tuberculínico foi positivo naqueles maiores de 21 anos, com cicatriz vacinal (BCG) e que não tiveram contato com indivíduo com tuberculose, $(p>0,05)$. Os estudantes que receberam a segunda dose de BCG tiveram maior chance de apresentar teste positivo quando confrontados com os que não foram vacinados $(R C=0,35$; $95 \%$ IC $=0,1-0,9 ; p=0,023)$. Conclusões. A maior prevalência de tuberculose latente ao final do curso médico sugere que os estudantes de medicina estão possivelmente expostos a transmissão nosocomial de Mycobacterium tuberculosis. Este estudo mostrou a vulnerabilidade desta população em relação à tuberculose e revelou a necessidade de implementação de programas de biossegurança para os estudantes de medicina, além de alertar para o uso de medidas de controle.

DESCRITORES: Teste tuberculínico; Tuberculose latente; Estudantes de medicina; Infecção nosocomial; Mycobacterium tuberculosis
ABSTRACT: Objectives. To verify the level of exposure to Mycobacterium tuberculosis and the associated risk factors, using the tuberculin test as a marker for latent tuberculosis in the university's medical school in UNIVÁS, Pouso Alegre, MG, Brazil. Methods. Cross-sectional study with 120 students divided into three groups: basic, intermediate and boarding. Information regarding gender, age, presence of BCG scar, the second dose of BCG, previous contact with tuberculosis patients and personal history of tuberculosis were obtained by questionnaire. The tuberculin test was performed by a skilled professional was defined as positive when the induration was greater than $5 \mathrm{~mm}$. Results. The prevalence of exposure to Mycobacterium tuberculosis was $28,3 \%$, being more frequent in the boarding school students. There was a greater positivity in females $(p>0.05)$. The tuberculin test was positive in those over 21 years, with the scars (BCG) and who had no contact with a person with tuberculosis $(p>0.05)$. Students who received the second dose of BCG were more likely to have positive test when compared with those not vaccinated $(\mathrm{RC}=0.35,95 \% \mathrm{Cl}=0.1$ to $0.9, p=0.023)$. Conclusions. The higher prevalence of latent tuberculosis at the end of medical school suggests that medical students are probably exposed to nosocomial transmission of Mycobacterium tuberculosis. This study showed the vulnerability of this population in relation to tuberculosis and revealed the need for implementation of biosafety programs for medical students and alerts for the use of control measures.

KEYWORDS: Tuberculin test; Latent tuberculosis; Medical students; Nosocomial infection; Mycobacterium tuberculosis

1. Mestre em Pediatria pela Faculdade de Ciências Médicas da Santa Casa de São Paulo, SP. Professor Auxiliar da Faculdade de Ciências Médicas da Universidade do Vale do Sapucaí/UNIVÁS, Pouso Alegre, MG.

2. Doutor e Mestre em Pneumologia pela Universidade Federal de São Paulo, Brasil. Professor Titular da Faculdade de Ciências Médicas da Universidade do Vale do Sapucaí/UNIVÁS, Pouso Alegre, MG.

3. Graduandos em Medicina, Faculdade de Ciências Médicas da Universidade do Vale do Sapucaí/UNIVÁS, Pouso Alegre, MG.

4. Residente de Clínica Médica, Hospital Universitário Samuel Libâneo, Universidade do Vale do Sapucaí/UNIVÁS, Pouso Alegre, MG.

Endereço para correspondência: Eugênio Fernandes de Magalhães. Rua Roberto Gonçalves Campos, 481, Pouso Alegre, MG, CEP: 37550-000. E-mail: magalhaeseugenio@ bol.com.br, efmaga@gmail.com 
Magalhães et al. Nível de exposição ao Mycobacterium tuberculosis em estudantes.

\section{INTRODUÇÃO}

A Organização das Nações Unidas tem como meta reduzir as taxas de incidência e de mortalidade pela tuberculose (TB) em 50\% até 2015. Dados de 2009 demonstram uma prevalência de 14 milhões de casos de TB sendo que 5,8 milhões foram notificados. A maioria dos casos foram na África, Ásia e algumas regiões do Pacífico'.

Apesar do Brasil ser um dos 22 países responsáveis por $90 \%$ dos casos de tuberculose do mundo, até o ano de 2007, ocorreu em nosso país uma queda de $26 \%$ na incidência e de $32 \%$ na mortalidade pela doença ${ }^{2}$.

Tanto os países desenvolvidos como os em desenvolvimento, os profissionais de saúdee estudantes representam um grupode risco importantepara a exposição, infecção e potencialmente doença $a^{3,4}$.

Muitos estudos apontam para maior risco entre os profissionais que atuam em estabelecimentos assistenciais de saúde, bem como estudantes da área da saúde e alertam que fatores com o tempo de serviço, categoria profissional e proteção respiratória são relevantes na transmissão $0^{5-8}$.

Além disso, outros trabalhos encontraram um aumento significativo de transmissão nosocomial, especialmente entre pacientes portadores de coinfecção HIV/TB e profissionais de saúde, quando as normas preconizadas de biossegurança não foram rigorosamente observadas ${ }^{9,10}$.

Os estudantes de medicina têm progressivamente maior contato com pacientes a partir da terceira série da faculdade de medicina, enquanto os alunos do curso básico não participam da assistência médica.

O Hospital das Clínicas Samuel Libâneo (HCSL), da Universidade do Vale do Sapucaí (UNIVÁS), em Pouso Alegre, é um hospital de referência para tuberculose no sul de Minas Gerais. É nele que os alunos do curso de medicina da UNIVÁS realizam as atividades práticas e treinamentos clínicos durante a graduação, estando expostos a um ambiente de elevado risco de contaminação pelo bacilo da tuberculose, onde são internados os pacientes com a infecção.

No ano de 2010 foram notificados 31 casos de TB que foram atendidos no HCSL ${ }^{11}$. É desconhecido o risco de infecção por Mycobacterium tuberculosis (MTb) no hospital que eles utilizam para seu treinamento.Diante disto, o presente estudo se propôs a verificar o nível de exposição ao MTbe os fatores de risco associados a ele, utilizando o teste tuberculínico (TT) como marcador para tuberculose latente (TL) em universitários do curso de medicina da UNIVÁS, seguindo a nova normatização na leitura do TT da III Diretrizes para Tuberculose da Sociedade Brasileira de Pneumologia e Tisiologia².

\section{MÉTODOS}

Foi realizado um estudo transversal em uma população de acadêmicos do curso de medicina da Universidade do Vale do Sapucaí (UNIVÁS) em Pouso Alegre, MG, no ano de 2010.

A universidade conta com um total de 3635 alunos sendo 1703 da área de biológicas, 1760 de humanas e 172 de exatas.

No curso de medicina, com duração de seis anos, ingressam 70 alunos anualmente. A pesquisa envolveu 120 estudantes que foram distribuídos de maneira uniforme entre os sexos e divididos em três grupos de 40 alunos, onde se denominou Grupo I para aqueles que estavam no ciclo básico do curso $\left(1^{\underline{a}} \mathrm{e}\right.$ $2^{\mathrm{a}}$ séries), Grupo II para aqueles no ciclo intermediário ( $3^{\mathrm{a}}$ e $4^{\mathrm{a}}$ séries) e Grupo III para aqueles que já frequentavam o internato médico ( $5^{\circ}$ e $6^{\circ}$ séries).

Para a coleta de dados foi utilizado um questionário autoaplicável, desenvolvido para a pesquisa, contendo perguntas relativas ao grupo, gênero, faixa etária, presença de cicatriz BCG, segunda dose de $B C G$, contato prévio com portador de tuberculose e história pessoal de tuberculose.

Os questionários foram aplicados pelos pesquisadores nas salas de aula após breve explicação da pesquisa e mediante assinatura de termo de consentimento livre e esclarecido, sem nenhuma identificação pessoal.

Cento e vinte acadêmicos foram escolhidos aleatoriamente e convidados a participar do trabalho. Após conclusão dos questionários, aplicou-se nos estudantes o TT com PPD Rt-23 (Derivado Protéico Purificado), utilizando-se a técnica de Mantoux.

Após 48 a 72 horas da administração do teste, os participantes retornaram para avaliação e medida da enduração.

A reação foi avaliada por uma equipe de enfermagem, que utilizaram o método da visualização, palpação, delineamento do contorno com caneta esferográfica e medida do diâmetro com régua milimetrada, padronizada pelo CDC. Denominou-se não reator aquele indivíduo com teste tuberculínico entre 0 e $4 \mathrm{~mm}$ e reator aquele com TT acima de $5 \mathrm{~mm}$, seguindo a norma das III Diretrizes para Tuberculose da Sociedade Brasileira de Pneumologia e Tisiologia².

Todos os alunos que apresentaram TT positivo foram encaminhados ao pneumologista para avaliação clínica, sendo solicitada radiografia de tórax e, quando indicado, baciloscopia de escarro.O uso de 
isoniazida no grupo com TL estaria indicado caso houvesse contato com grupos especiais, viragem tuberculínica e radiografia de tórax normal ${ }^{2}$. O estudo foi aprovado pelo Comitê de Ética em Pesquisa da Faculdade de Ciências da Saúde Dr. José Antônio Garcia Coutinho, da Universidade do Vale do Sapucaí (UNIVÁS). Na análise estatística de comparação de variáveis entre os sexos, fatores de risco e moradia utilizou-se o Teste do Qui-quadrado ${ }^{12}$ para confrontar os grupos de respostas com diferentes variáveis. Para cada variável de interesse, foi calculado o valor do odds-ratio e seu respectivo intervalo de confiança. Foram consideradas significativas as diferenças que apresentaram $p<0,05$.

\section{RESULTADOS}

No presente estudo houve a participação efetiva de 120 alunos do curso de medicina que foram escolhidos aleatoriamente, com taxa de adesão de $100 \%$. Todos os alunos que foram submetidos ao teste foram reavaliados, não havendo perdas. Dentre eles, $60(50 \%)$ eram do gênero masculino e $60(50 \%)$ do feminino. A prevalência global de expostos ao MTb foi de $28,3 \%$ ( $p>0,05)$, naqueles reatores com TT acima de $5 \mathrm{~mm}$. Quando segmentamos os grupos, a prevalência de reatores fortes foi de $22,5 \%, 30 \%$ e 32,5\% nos Grupos I, II e II, respectivamente, não havendo diferença estatisticamente significativa entre eles. Durante a estratificação por gênero, quando se avaliou os testes positivos, a prevalência foi maior nos alunos (25\%) do Grupo I e nas alunas (40\%) do Grupo III (Tabela 1).

Tabela 1. Resultados do teste tuberculínico nos alunos do curso de medicina dos Grupos I, II e III, Pouso Alegre (MG), 2010

\begin{tabular}{|c|c|c|c|c|}
\hline Variáveis & $\begin{array}{c}\text { TT negativo } \\
\mathrm{N}(\%)\end{array}$ & $\begin{array}{c}\text { TT positivo } \\
\mathrm{N}(\%)\end{array}$ & $\begin{array}{l}\text { Total } \\
\text { N (\%) }\end{array}$ & $\mathbf{P}$ \\
\hline $\begin{array}{c}\text { Grupo I } \\
\text { Masculino } \\
\text { Feminino } \\
\text { Total }\end{array}$ & $\begin{array}{r}15(75) \\
16(80) \\
31(77,5)\end{array}$ & $\begin{array}{r}5(25) \\
4(20) \\
9(22,5)\end{array}$ & $\begin{array}{l}20(100) \\
20(100) \\
40(100)\end{array}$ & $p>0,999$ \\
\hline $\begin{array}{l}\text { Grupo II } \\
\text { Masculino } \\
\text { Feminino } \\
\text { Total }\end{array}$ & $\begin{array}{r}14(70) \\
14(70) \\
28(70)\end{array}$ & $\begin{array}{r}6(30) \\
6(30) \\
12(30)\end{array}$ & $\begin{array}{l}20(100) \\
20(100) \\
40(100)\end{array}$ & $p>0,999$ \\
\hline $\begin{array}{c}\text { Grupo III } \\
\text { Masculino } \\
\text { Feminino } \\
\text { Total }\end{array}$ & $\begin{array}{r}15(75) \\
12(60) \\
27(67,5)\end{array}$ & $\begin{array}{r}5(25) \\
8(40) \\
13(32,5)\end{array}$ & $\begin{array}{l}20(100) \\
20(100) \\
20(100)\end{array}$ & $p=0,501$ \\
\hline
\end{tabular}

Na Tabela 2 durante a análise bivariada observou-se, quanto ao gênero, uma maior positividade no sexo feminino $(p>0,05)$. O teste tuberculínico foi positivo nos alunos maiores de 21 anos, com cicatriz vacinal (BCG) e naqueles que não tiveram contato com indivíduo com a Tuberculose, sem significância estatística. Os estudantes que receberam a segunda dose de BCG tiveram maior chance de apresentar TT positivo quando confrontados com os que não foram vacinados $(R C=0,35 ; 95 \% I C=0,1-0,9$; $p=0,023)$.

\section{DISCUSSÃO}

Demonstrou-se nesta pesquisa que a prevalência de expostos ao MTb através do TT positivo foi de $28,3 \%$ sendo maior no grupo III. O TT foi positivo em sua maioria no gênero feminino, naqueles maiores de 21 anos, com cicatriz de BCG e que receberam a segunda dose. A maior parte dos alunos com historia de contato com paciente com TB não reagiram ao teste tuberculínico. Naqueles países onde houve o ressurgimento da TB e o aparecimento de altos índices de resistência a múltiplas drogas pelo bacilo, o risco de tuberculose latente e tuberculose doença aumentou significativamente entre os profissionais de saúde ${ }^{13}$. Apesar das diretrizes da OMS sobre a TB nas instituições de saúde, não existe nenhuma normatização a respeito das atividades a serem implementadas em cursos de graduação na área da saúde ${ }^{14}$. Ferreira ${ }^{15}$, Silva ${ }^{16}$ e Costa ${ }^{17}$ mostraram prevalência de TT positivos de $20,4 \%, 9,2 \%$ e $3,6 \%$, respectivamente, entre estudantes de medicina, em estudos realizados em hospitais universitários nas cidades do Rio de Janeiro (RJ) e Niterói (RJ). Estudo envolvendo alunos de várias escolas médicas 
Magalhães et al. Nível de exposição ao Mycobacterium tuberculosis em estudantes.

do Rio de Janeiro mostrou uma prevalência de TB latente de 6,9\% ${ }^{13}$. Maciel ${ }^{18}$ em 1999 constatou que dos 178 estudantes de enfermagem da Universidade Federal do Espírito Santo, 39,4\% apresentaram-se reatores ao teste tuberculínico, sendo $25,3 \%$ reatores fortes e $14 \%$ reatores fracos. Desses, $33,3 \%$ pertenciam ao primeiro ano de faculdade; portanto, sem ter ainda entrado em contato com pacientes com TB na faculdade. Entretanto, desse percentual, 42\% relataram pertencer à outra categoria profissional (técnico, auxiliar de enfermagem ou de laboratório). Quadros-Coelho ${ }^{19}$ demonstrou que 18,2\% e37\% dos estudantes dos primeiros e dos últimos períodos de medicina foram, respectivamente, fortes reatores ao teste tuberculínico, corroborando com nosso estudo. Esses dados sugerem que a exposição ao MTb em estudantes de medicina no nosso país, com alta endemicidade de TB, aumenta de acordo com a maior exposição ao MTb durante seus anos do curso médico.

Tabela 2. Resultados da análise bivariada dos fatores curriculares e extra-curriculares associados com o teste tuberculínico positivo entre os alunos de medicina, Pouso Alegre (MG), 2010

\begin{tabular}{|c|c|c|c|c|c|}
\hline Variáveis & $\begin{array}{c}\text { TT negativo } \\
\mathrm{N}(\%)\end{array}$ & $\begin{array}{c}\text { TT positivo } \\
\mathrm{N}(\%)\end{array}$ & $\mathrm{RC}$ & IC & $\mathbf{P}$ \\
\hline \multicolumn{6}{|l|}{ Sexo } \\
\hline Masculino & $44(73,3)$ & $16(26,7)$ & 1,2 & $(0,5-2,6)$ & $p=0,685$ \\
\hline Feminino & $42(70)$ & $18(30)$ & & & \\
\hline \multicolumn{6}{|l|}{ Faixa etária } \\
\hline$<21 \mathrm{a}$ & $25(75,8)$ & $8(24,2)$ & 1,3 & $(0,5-3,3)$ & $p=0,541$ \\
\hline$>21 \mathrm{a}$ & $61(70,1)$ & $26(29,9)$ & & & \\
\hline \multicolumn{6}{|l|}{ Cicatriz BCG } \\
\hline $\operatorname{Sim}$ & $83(70,9)$ & $34(29,1)$ & 0,9 & $(0,9-1,1)$ & $p>0,999$ \\
\hline Não & $3(100)$ & $95(0)$ & & & \\
\hline \multicolumn{6}{|l|}{$2^{a}$ dose $B C G$} \\
\hline Sim & $46(63,9)$ & $26(11,6)$ & 0,35 & $(0,1-0,9)$ & $p=0,023$ \\
\hline Não & $40(83,3)$ & $8(16,7)$ & & & \\
\hline \multicolumn{6}{|c|}{ Contato com paciente com TB } \\
\hline Sim & $9(81,8)$ & $2(18,2)$ & 1,9 & $(0,4-9,1)$ & $p=0,439$ \\
\hline Não & $77(70,6)$ & $32(29,4)$ & & & \\
\hline \multicolumn{6}{|c|}{ Histórico pessoal de TB } \\
\hline $\operatorname{Sim}$ & $0(0)$ & $0(0)$ & & & \\
\hline Não & $86(71,7)$ & $34(28,3)$ & & & \\
\hline
\end{tabular}

A prevalência elevada observada em nosso estudo pode ser atribuída, entre outras causas, ao fato de seguir nova normatização na leitura do TT da III Diretrizes para Tuberculose ${ }^{2}$, que pode ampliar o número de fortes reatores. Cerca de dois terços de nossa amostra não estava infectada pelo MTb, assim como a de Ferreira ${ }^{15}$ e a de Silva ${ }^{16}$, o que indica uma proporção relativamente elevada de alunos ainda não expostos ao MTb, exercendo atividades no HCSL/UNIVÁS, e portanto, estarão sob elevado risco de se infectarem pelo bacilo da tuberculose, caso não se incentive o uso de medidas de biossegurança apropriadas. O gênero feminino teve uma proporção maior de TL, como demonstrado por outros autores ${ }^{7,20}$. Nossos resultados corroboram os estudos de Soares ${ }^{7}$, Teixeira ${ }^{14}$ e Muñoz-Barret ${ }^{21}$ onde se observou associação entre TT positivo e vacinação com BCG, verificada pela sua cicatriz. Sabe-se que a maioria dos participantes do nosso estudo foi vacinada quando criança. No nosso trabalho, a positividade ao TT provavelmente não deve ser devida à vacinação pelo BCG. Assim, o TT continua útil na avaliação de infecção nosocomial por MTb. Os alunos que receberam a $2^{\underline{a}}$ dose de BCG tiveram prevalência significativamente maior de TT positivo quando confrontados com aqueles não foram revacinados. É importante ressaltar que não existem evidências para a $2^{a}$ dose da BCG, suspensa pelo Ministério da Saúde em 2006². Vários estudos sobre revacinação BCG não mostraram proteção conferida pela segunda dose da vacina nos adolescentes e profissionais de saúde $22-24$. O estudo não mostrou 
associação entre a TT positivo e contato com paciente com TB se contrapondo aos dados encontrados em outras pesquisas ${ }^{8,14}$. O teste ideal para diagnóstico de TL deveria ter altas sensibilidade e especificidade, independentemente de história prévia de vacinação com BCG e infecção por outras micobactérias. $O$ TT, que vem sendo por quase um século o método utilizado como padrão-ouro na identificação desses pacientes, é um teste in vivo que avalia a reação de hipersensibilidade cutânea tardia, utilizando um PPD como antígeno. Uma alternativa para o diagnóstico de TL, atualmente feito pelo TT, são os chamados IGRAS (interferon-gamma release assays). Tais testes in vitro avaliam a produção dessa citocina por células previamente sensibilizadas com agentes específicos $^{25}$. Dentre as vantagens, os IGRAs parecem não ser influenciados pela vacinação prévia com BCG ${ }^{26}$, necessidade de apenas uma visita (sem retorno para a leitura, como no TT), resultados em 24 horas, eliminação de erros de aplicação e interpretação de leitura do TT, uso de pequeno volume de sangue e ausência do efeito booster, por não expor o próprio paciente a um antígeno ${ }^{27}$. Zwerling et al. ${ }^{28}$ concluiram que o uso de IGRAs, ao invés do TT para uma triagem unica, pode resultar emuma prevalênciamenor deTT positivoe uma quantidade menor de profissionais de saúde que requeiram tratamento de TL, particularmente em locais onde a incidência de TB é baixa. Por outro lado, o uso de IGRAs ainda não está validado para o uso de rotina no Brasil2 ${ }^{2}$ Nosso estudo teve algumas limitações. Apesar de termos o número de alunos previsto na amostra, houve um viés de seleção no estudo, já que a escolha deles foi aleatória e participação opcional, já que não existem mecanismos que possibilitem a inclusão obrigatória de alunos nesse tipo de estudo. Há uma chance de que alunos já sabidamente reatores à TT tenham evitado participar do estudo e de aqueles com exposição prévia a doentes com TB tenham se interessado mais em participar, como demonstrado em outros estudos ${ }^{14,16}$. Também deve ser levado em consideração a ausência de realização de um segundo teste tuberculínico duas a três semanas após o TT para avaliar o fenômeno booster. Haja vista que o Centers for Diseases Control and Prevention dos EUA (CDC) recomendam que quando um teste periódico é realizado como acompanhamento anual do pessoal hospitalar, os indivíduos que responderam negativamente ao teste cutâneo inicial devem repeti-lo uma semana após o primeiro teste. Esse procedimento evita a classificação de falsos negativos ${ }^{29}$. Os estudos transversais têm relativa limitação na capacidade de indicar associações causais. Um estudo longitudinal seria mais apropriado para avaliação de risco de infecção, taxa de conversão e fatores associados ao risco de infecção.

\section{CONCLUSÃO}

Concluiu-se, através dos resultados obtidos, que os estudantes de medicina são possivelmente expostosa transmissão nosocomial de MTb, sugerido pelo aumento da prevalência de reatores à tuberculina entre os alunos no final de estudos médicos. Entretanto, com a crescente tendência de haver maior proporção de infectados no decorrer dos períodos, levantou-se a hipótese de estar ocorrendo viragem tuberculínica neste tempo de práticas ambulatoriais e hospitalares mais frequentes, como demonstrado por outros autores ${ }^{15,17}$. Os dados da literatura sugerem que a incidência da infecção pelo Mycobacterium tuberculosis em estudantes de medicina, não difere de países com baixa incidência da doença ${ }^{30,31}$. Este estudo mostrou a vulnerabilidade desta população em relação à $T B$ e revelou a necessidade de implementação de programas de biossegurança para os estudantes de medicina, além de alertar para o uso de medidas de controle, incluindo isolamento respiratório de casos confirmados ou suspeitos de TB e a proteção respiratória individual durante $o$ atendimento ao paciente infectado. Devese avaliar o uso rotineiro do TT nos estudantes de medicina para identificar TB latente, principalmente nos ingressantes. Diante disso, seria interessante a indicação de quimioprofilaxia, quando houvesse viragem tuberculínica durante o curso médico.

Agradecimentos: A todos os estudantes dos cursos de medicina que participaram deste estudo; à bibliotecária Lucilene Marques, pela revisão bibliográfica; à professora Cremilda Eufrásio, que nos auxiliou na leitura do teste tuberculínico e à Fundação de Amparo a Pesquisa de Minas Gerais (FAPEMIG).

\section{REFERÊNCIAS}

1. Global tuberculosis control. WHO report 2010. Geneva, Switzerland: WHO Press; 2010. Avaliable from: http://www.whqlibdoc.who.int/ publications/2010/9789241564069_eng.pdf.

2. Conde MB, Melo FAF, Marques AMC, Cardoso NC, Pinheiro VGF, Dalcin PTR, et al. III Diretrizes para
Tuberculose da Sociedade Brasileira de Pneumologia e Tisiologia. J Bras Pneumol. 2009;35(10):1018-48.

3. Joshi R, Reingold AL, Menzies D, Pai M. Tuberculosis among health-care workers in low- and middleincome countries: a systematic review. PLoS Med. 2006;3:e494. 
4. Menzies D, Fanning A, Yuan L, Fitzgerald M. Tuberculosis among health care workers. $\mathrm{N}$ Engl $\mathrm{J}$ Med. 1995;332:92-98.

5. Oliveira SM, Honner MR, Paniago AM, Aguiar ES, Venâncio da Cunha R. Prevalence of Mycobacterium tuberculosis among professionals in a university hospital, Mato Grosso do Sul, 2004. Rev Lat-Am Enferm. 2007;15(6):1120-4.

6. Vendramini SHF, Villa TCS, Santos MLSG, Geraldes MSC. Aspectos epidemiológicos atuais da tuberculose e o impacto da estratégia DOTS no controle da doença. Rev Latino-Am Enferm. 2007; 15(1):171-3.

7. Soares LC, Mello FC, Kritski AL. Prevalência de prova tuberculínica entre alunos da Faculdade de Medicina de Campos (RJ). J Bras Pneumol. 2004;30(4):440-7.

8. Silva VM, Oliveira JR, Santos FM, Araújo CM, Kritski AL. Prevalência de infecção pelo Mycobacterium tuberculosis entre alunos da Faculdade de Medicina da Universidade Federal do Rio de Janeiro. J Bras Pneumol. 2001;27(2):77-82.

9. Sokolove PE, Mackey D, Wiles J, Lewis RJ. Exposure of emergency department personnel to tuberculosis: PPD testing during an epidemic in the community. Ann Emerg Med. 1994;24(3):418-21.

10. Zaza S, Blumberg HM, Beck-Sagué C, Haas WH, Woodley CL, Pineda M, et al. Nosocomial transmission of Mycobacterium tuberculosis: role of health care workers in outbreak propagation. J Infect Dis. 1995;172(6):1542-9.

11. Brasil. Ministério da Saúde. Portal da saúde: tuberculose. Avaliable from: http://dtr2004.saude.gov. $\mathrm{br} /$ sinanweb.

12. Siegel S. Estadística no paramétrica aplicada a las ciencias de la conducta. México: Trillas; 1975.

13. Ratio M, Helenius H, Tala E. Is the risk of occupational tuberculosis higher for young health care workers? Int J Tuberc Lung Dis. 2003;7(2):556-62.

14. Teixeira EG, Menzies D, Comstock GW, Cunha AJ, Kristki AL, Soares LC. Latent tuberculosis infection among under-graduate medical students in Rio de Janeiro State, Brazil. Int J Tuberc Lung Dis. 2005;9(5):841-7.

15. Ferreira FM. Prevalência de infecção por Mycobacterium tuberculosis em estudantes de medicina da Universidade do Rio de Janeiro UNI-RIO [tese]. Rio de Janeiro: UFRJ; 1998.

16. Silva VMCS. Prevalência da infecção pelo Mycobacterium tuberculosis, entre alunos de graduação da Faculdade de Medicina e entre alunos de áreas não relacionadas à saúde, na Universidade Federal do Rio de Janeiro [dissertação]. Rio de Janeiro: UFRJ; 1998.

17. Costa PA. Avaliação da infecção tuberculosa em internos de medicina da Universidade Federal Fluminense [dissertação]. Niterói: Universidade Federal Fluminense; 2000.

18. Maciel EL. Infecção por Mycobacterium tuberculosis em estudantes de enfermagem: um estudo de incidência através do Teste PPD [dissertação]. Rio de Janeiro: UFRJ; 1999.
19. Quadros-Coelho MA, Biscotto CR, Tinois BR, Freitas FC, Oliveira JS, Azevedo MF, et al. Prevalência da infecção tuberculosa em universitários da Universidade Estadual de Montes Claros, Minas Gerais. Rev Bras Educ Med. 2009;33(4):535-41.

20. Xu Y, Schwartzman K. Referrals for positive tuberculin tests in new health care workers and students: a retrospective cohort study. BMC Public Health. 2010;10:28.

21. Muñoz-Barret JM, Macias-Hernandez AE, HernandezRamos I. Comparative tuberculin reactivity to two protein derivatives. Rev Invest Clin. 1996;48:377-81.

22. Rodrigues LC, Pereira SM, Cunha SS, Genser $B$, Ichihara MY, de Brito SC, et al. Effect of BCG revaccination on incidence of tuberculosis in schoolaged children in Brazil: the BCG-REVAC clusterrandomised trial. Lancet. 2005;366(9493):1290-5.

23. Pereira SM, Dantas OMS, Ximenes R, Barreto ML. Vacina BCG contra tuberculose: efeito protetor e políticas de vacinação. Rev Saude Publica. 2007;41(Suppl1):59-6.

24. Brasil. Ministério da Saúde. Manual de recomendações para o controle da tuberculose no Brasil. Brasília: Ministério da Saúde; 2010. Avaliable from: http:// portal.saude.gov.br/portal/arquivos/pdf/manual_de recomendacoes_controle_tb_novo.pdf.

25. Sztajnbok FR, Boechat NL, Sztajnbok DC, Brumibeiro S, Oliveira SK, Sant'Anna CC. The challenge of pediatric tuberculosis in face of new diagnostic techniques. J Pediatr.2009;85:183-93.

26. Ferrara G, Losi M, Meacci M, Meccugni B, Piro $R$, Roversi $P$, et al. Routine hospital use of a new commercial whole blood interferon gamma assay for the diagnosis of tuberculosis infection. Am J Respir Crit Care Med. 2005;172:631-5.

27. Mazurek GH, Jereb J, Vernon A, LoBue P, Goldberg S, Castro K. Update guideline for using interferon gamma release assays to detect Mycobacterium tuberculosis infection - United States, 2010. MMRW Recomm Rep. 2010;59(RR-5):1-28.

28. Zwerling A, van den Hof S, Scholten J, Cobelens F, Menzies D, Pai M. Interferon-gamma release assays for tuberculosis screening of healthcare workers: a systematic review. Thorax. 2011 [Epub ahead of print].

29. Centers for Disease Control and Prevention. Guidelines for preventing the transmission of $M$. tuberculosis in health care facilities. MMWR Recomm Rep.1994;43(RR-13):1-132.

30. Maciel EL, Meireles W, Silva AP, Fiorotti K, Dietze R Nosocomial Mycobacterium tuberculosis transmission among health care students in a high incidence setting, Vitoria, ES. Rev Soc Bras Med Trop. 2007;40(4):3979.

31. Esteban J, Gadea I, Fernández Roblas R, Granizo JJ, Soriano F. Tuberculous infection in student nurses in Madrid, Spain. Int J Tuberc Lung Dis. 2001;5:583-4.

Artigo recebido em: 10/01/2011

Artigo aceito em: 11/04/2011 